\title{
Miniaturized System with a Facile Microfluidic Chip for DNA Extraction, Isothermal Amplification and Fluorescence Detection
}

\author{
Xing Chen, Lulu Zhang, Dafu Cui \\ State Key Laboratory of Transducer Tech., Institute of Electronics, Chinese Academy of Sciences, \\ Beijing, 100190, China \\ Corresponding author's e-mail address : xchen@mail.ie.ac.cn
}

\begin{abstract}
:
In this paper, a biomedical miniaturized system with a disposable microfluidic chip has been developed for solid phase extraction (SPE), loop-mediated isothermal amplification (LAMP) and realtime fluorescence detection. DNA was extracted from real biological samples, such as whole blood, by using a Glass-polydimethylsiloxane (PDMS)-glass microfluidic chip with a cell, which were also designed and fabricated for loop-mediated isothermal amplification (LAMP) in the same cell. LAMP reaction and real-time fluorescence detection were implemented by using the miniaturized system including a mini heating plate, a temperature sensor, a temperature controller, fluorescence detector and a adjustable mechanical stage. DNA has successfully extracted from whole blood and then amplification and detection. This biomedical system has the potential for point-of-care diagnostics (POCT) with the many advantages, such as not high cost, short analysis and detection time, not much reagent and sample consumption and so on.
\end{abstract}

Key words: Microfluidic chips, Loop-mediated Isothermal Amplification (LAMP), Solid Phase Extraction (SPE), Miniaturized System, Fluorescence Detection.

\section{Introduction}

With the development of microfluidics technology, many efforts have been focused on lab-on-a-chip or micrototal analysis systems. Lab-on-a-chip or micrototal analysis systems have some advantages like high throughput, short analysis time, small volumes and high sensitivities [1], which can be capable of ready measurement of disease biomarkers in physiological fluids [2,3].

Combination of nucleic acid analysis technique and microfluidic technology will facilitate the realization of point-of-care (POC) pathogen detection. Uddin et. al. [4] reported a portable end-point detection system for LAMP reactions on microfluidic compact disk platform, which could lead to the development of a point-of-care diagnosis device for foodborne pathogens detection in a resource-limited environment. However, there was lack of sample prepare step, such as DNA extraction step.

In this paper, an integrated system has been developed to extract DNA, amplified and detect the amplification products in real-time for the point-of-care test (POCT) .

\section{Experimental section}

Microfluidic chips were designed and fabricated with several microcells by using PDMS, shown in Fig 1.

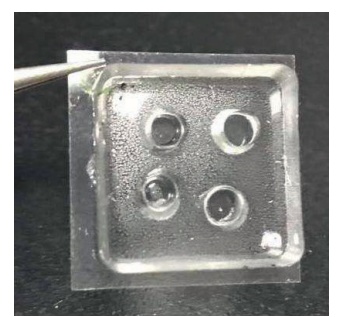

Fig. 1. Photograph of the microfluidic chip with four cells for DNA extraction, isothermal Amplification and real-time fluorescence detection.

Before LAMP reactions, different samples with or without the positive amplification template were used for DNA extraction step by using a normal pipette. The DNA extraction procedure itself consisted of load, wash, elution steps (details in our previous research [5]). $20 \mu \mathrm{L}$ load buffer (4M NaCl in TE, pH 6.4) containing $1 \%$ Triton X-100 and $5 \mu \mathrm{L}$ rat whole blood with or without $1 \mu \mathrm{L}$ positive amplification template were dropped into different cells, respectively. 
DNA was binding onto bottom of the cell. Proteins and possible inhibitors that were adsorbed onto the bottom during load step were removed by using wash buffer (70 \% ethanol) twice. And then $10 \mu \mathrm{L}$ Isothermal Master Mix (Optigene, the United Kingdom) containing $0.125 \mathrm{nM}$ each of the outer primers F3 and B3, $1.0 \mathrm{nM}$ each of the primers FIP and BIP, and $0.5 \mathrm{nM}$ each of the primers LF and LB was dropped into the cells. Finally, the microchip with the cells was put on our miniaturized system for isothermal amplification and fluorescence detection.

Several microliters of liquid paraffin and low temperature paraffin were added to avoid evaporation during heating process. The amplification reactions in microchips were performed at $60{ }^{\circ} \mathrm{C}-65^{\circ} \mathrm{C}$ in our miniaturized system for $1 \mathrm{~h}$.

\section{Results and conclusion}

The miniaturized system was developed consisting of a mini heating plate, a $\mathrm{Pt}$ temperature sensor, a PID temperature controller, a adjustable mechanical stage and a portable confocal fluorescent detector (ESE LOG ESML 10-MB-3007, Germany) shown in Fig. 2. This system with a microfluidic chip was used to implement the LAMP reaction and the real-time fluorescent detection of the amplification products. The results of LAMP procedure was output from the computer.

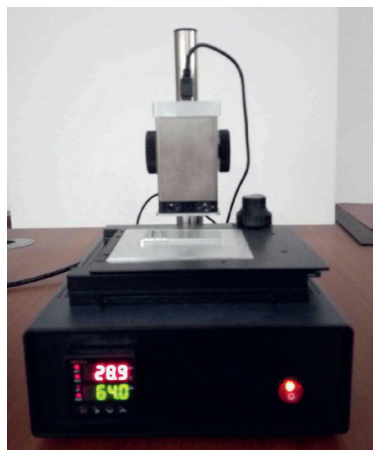

Fig. 2. Photograph of the miniaturized system for real-time detection of $L A M P$ reaction on a microfluidic chip.

Different rat whole blood samples with or without the amplification template were used for DNA extraction, LAMP reaction and real-time fluorescence detection. The results of experiments are shown in Fig. 3. From the dynamic cures of the real-time fluorescence detection, DNA has been successfully extracted, amplified and detected by using one microfluidic chip.
This biomedical system with the microchip has the potential for point-of-care diagnostics including the sample pre-treatment step.

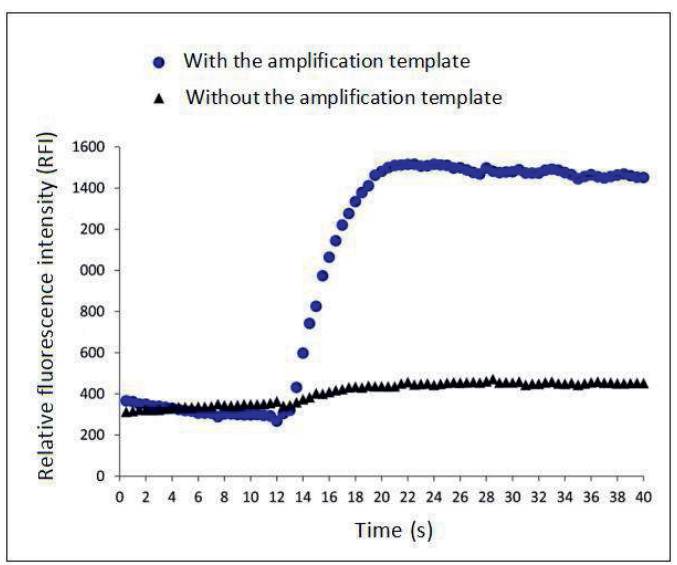

Fig. 3. Dynamic curves of the real-time fluorescent detection of the amplification products of LAMP reactions with or without the amplification template on microfluidic chips by using our miniaturized system.

\section{Acknowledgements}

The authors greatly acknowledge the financial support from the Major National Scientific Research Plan under Grant number 2014CB744603 and the National Science Foundation of China under Grant number 31571007,61571420 and 61372055.

\section{References}

[1] A. Arora, G. Simone, G.B. Salieb-Beugelaar, Latest developments in micro total analysis systems, Analytical Chemistry 82 (2010) 48304847.

[2] B. S. Ferguson, S. F. Buchsbaum, T. T. Wu, Genetic analysis of H1N1 influenza virus from throat swab samples in a microfluidic system for point-of-care diagnostics, Journal of the American Chemical Society, 133(2011)9129-9135.

[3] S. Lingam, M. Beta, D. Dendukuri, A focus on microfluidics and nanotechnology approaches for the ultra sensitive detection of microRNA, Microrna, 3(2014)18-28

[4] S. M. Uddin, F. Ibrahim, A. A. Sayad, A Portable Automatic Endpoint Detection System for Amplicons of Loop Mediated Isothermal Amplification on Microfluidic Compact Disk Platform, Sensors, 15(2015)5376-5389.

[5] X. Chen, D. Cui., J. Sun, L. Zhang, H. Li, Microdevice-based DNA Extraction Method Using Green Reagent, Key Engineering Materials, 562565 (2013) 1111-1115 